\title{
Modelo Logit para la Presencia de Problemas Osteomusculares en Trabajadores del Sector Hospitalario
}

\author{
Elías A. Bedoya-Marrugo ${ }^{(1)^{*}}$, Luz E. Vargas-Ortiz ${ }^{(2)}$, Carlos A. Severiche-Sierra ${ }^{(1)}$ y María de J. Meza-Aleman ${ }^{(1)}$ \\ (1) Fundacion Universitaria Tecnologico Comfenalco - Cartagena, Facultad de Ingeniería, Programa de \\ Seguridad e Higiene Ocupacional, grupo CIPTEC, Cartagena de indias, Bolívar, Colombia \\ (e-mail: ebedoya@tecnologicocomfenalco.edu.co). \\ (2) Fundacion Universitaria Tecnologico Comfenalco - Cartagena, Facultad de Ingeniería, Programa de \\ Ingeniería Industrial, Cartagena de indias, Bolívar, Colombia \\ ${ }^{*}$ Autor a quien debe ser dirigida la correspondencia
}

Recibido Jun. 27, 2018; Aceptado Sep. 10, 2018; Versión final Nov. 30, 2018, Publicado Abr. 2019

\begin{abstract}
Resumen
El objetivo de este trabajo es identificar condiciones sociodemográficas y laborales asociadas a problemas osteomusculares en trabajadores del sector hospitalario. Se realizó un Modelo Logit para determinar las variables sociodemográficas y laborales que influyen en la presencia de problemas osteomusculares en un grupo de 123 trabajadores del sector salud. Para el análisis de los resultados se calcularon estadísticas descriptivas, se realizó un diagrama de Pareto, y se ajustó un modelo Logit. La variable respuesta corresponde a la presencia de problemas osteomusculares y se realizaron gráficos de mosaico entre la presencia de problemas osteomusculares y las variables que resultaron significativas en el modelo. Los resultados indican que los problemas osteomusculares de mayor presencia son problemas en las manos y dolor en los huesos y el Modelo Logit muestra que el género, la antigüedad laboral y la responsabilidad del cargo influyen en la presencia de problemas osteomusculares.
\end{abstract}

Palabras clave: Modelos Logit; personal de salud; salud laboral; trastornos musculares.

\section{Logit Model for the Presence of Osteomuscular Problems in Workers of the Healthcare Sector}

\begin{abstract}
The objective of this work is to identify sociodemographic and work conditions associated with musculoskeletal problems in workers in the hospital sector. A Logit Model was used to determine the sociodemographic and labor variables that influence the presence of musculoskeletal problems in a group of 123 workers in the health sector. For the analysis of the results, descriptive statistics were calculated, a Pareto diagram was made, and a Logit model was adjusted. The response variable corresponds to the presence of musculoskeletal problems and mosaic charts were made between the presence of musculoskeletal problems and the variables that were significant in defining the model. The results indicate that the musculoskeletal problems of greater presence are hand problems and bone pain and the Logit Model shows that gender, seniority and the responsibility position influence the presence of musculoskeletal problems.
\end{abstract}

Keywords: Logit models, health personnel, occupational health, muscular disorders. 


\section{INTRODUCCIÓN}

En el campo hospitalario se han referenciado diversos factores de riesgo ocupacional con ejercicios, esfuerzos, frecuentes y extremos, lo que puede conducir al desarrollo de dolor lumbar (Bedoya et al., 2018a; Bedoya et al., 2018b; Nourollahi et al., 2018; Bedoya et al., 2017a; Wang et al., 2015; Yasobant y Rajkumar, 2014). Personal de ciencias del cuidado conformado por 1.396, principalmente mujeres han informado más de 1 síntoma donde el dolor lumbar afectó al $88 \%$ de estas, siendo además la queja más prevalente con $60.9 \%$. La ocurrencia de estas tareas tuvo lugar en más de 10 ocasiones al día, con paciente en la cama (Burgos et al., 2018; Afshari et al., 2014). Dentro del personal expuesto, los riesgos potenciales para las enfermeras está el realizar movimientos frecuentes y de gran amplitud del tronco, lo que puede contribuir al desarrollo de trastornos de la parte baja de la espalda y molestias en extremidades superiores (Bedoya et al., 2018c; Bedoya et al., 2017b; Szeto et al., 2013). Por otra parte, se han consultado fuentes que indican que los problemas osteomusculares en el trabajo se han consolidado como verdaderas dificultades de salud pública en todo el mundo, afectando indistintamente a hombres y mujeres en el servicio hospitalario, siendo actualmente considerados como trastornos laborales, que afectan en especial, a encargados de labores propias de la enfermería (Homaid et al., 2016), lo cual sumado a la escasa mano de obra, la sobrecarga laboral de las mismas, incrementan la carga de lesiones y discapacidades relacionadas, registros clínicos de fisioterapia señalan que las consultas por problemas osteomusculares han aumentado considerablemente, aunque la prevalencia y los factores asociados con este fenómeno no están claros (Dlungwane et al., 2018).

Otro estudio realizado ha permitido una cifra aproximada de la afectación en relación con los problemas osteomusculares donde se pudo afirmar que más del $80 \%$ de los profesionales de enfermería en Uganda, han presentado quejas y solicitudes de atención medica ocupacional al sentir afectaciones en diversas partes del cuerpo, en especial la zona lumbar, mostrando factores de riesgo predisponentes para esta condición el estar ausente de la estación de trabajo durante más de 6 meses debido a una enfermedad o un accidente, trabajar en posturas incómodas, empujar / jalar cargas pesadas, con lo cual se menciona la necesidad de mayor promoción, mejores condiciones de trabajo y la adopción de estrategias para reducir las lesiones laborales (Munabi et al., 2014). Algunos eventos de riesgo biomecánico y padecimientos relacionados tienen aparición repentina y duración de más de un día, cronicidad indeterminada se muestran como afecciones importantes en grupos significativos de trabajadores del sector salud (Bedoya et al., 2017c; Nodooshan et al., 2016). Existe asociación con otros factores desencadenantes como algún grado de obesidad, el historial de lesiones previas y la ejecución de trabajos de alta exigencia (Bedoya et al., 2018d; Yusoff et al., 2017). En otros estudios se ha detectado una alta incidencia de dolor lumbar entre las enfermeras como manifestaciones de trastornos musculoesqueléticos algunos sin carácter crónico y otros con indicios laborales (Bedoya et al., 2017d; Harcombe et al., 2014).

Resultados de estudios en áreas asistenciales de ginecología y obstetricia demuestran que el trastorno osteomuscular es alto al $85.5 \%$ donde el segmento corporal más afectado son los hombros $(n=575,62.0 \%)$, el cuello $(n=560,60.3 \%)$ y la zona lumbar $(n=504,54.3 \%)$ (Wang et al., 2017). En el mismo sentido Guimarães y Felli (2016), confirman a los factores individuales, posturales, trabajo-medioambientales y psicosociales como posibles generadores del daño. En orden de importancia las morbilidades más frecuentes y según los estudios de Balasubramanya et al. (2016) entre enfermeras, aparecen la diabetes mellitus (11\%), hipertensión (10,6\%), trastornos musculoesqueléticos $(9,3 \%)$ y eventos quirúrgicos $(8.6 \%)$ como las principales quejas de enfermedad detectadas. Para Weiner et al. (2015), el reposicionamiento de los pacientes por parte de los trabajadores asistenciales exige en estos últimos esfuerzos que son detonantes de lesión osteomuscular. Asimismo según lo realizado por Alperovitch-Najenson et al. (2014), los mencionados ejercicios incrementan el riesgo de sufrir lesiones ocupacionales. Estas se atribuyen a las demandas físicas excesivas en las enfermeras debido al peso de los pacientes y las posturas incómodas de la enfermera. Finalmente para Yan et al. (2017), la prevalencia de afecciones osteomusculares en el trabajo oscilan entre $81.18 \%$ y $77.43 \%$, entre enfermeras activas, con menor afectación en hombres, donde existió daño en dos o más regiones corporales como la zona lumbar, el cuello, el hombro y la espalda, existiendo bajas e incapacidades por enfermedad debida a molestias osteomusculares de principalmente asociadas con síntomas en la espalda $(6.17 \%)$, cuello $(3.06 \%)$, tobillo $(2.10 \%)$ y rodilla $(1.68 \%)$.

Altas proporciones de personal asistencial en especial enfermeras reportan anualmente dolores en algún sitio del cuerpo, siendo la espalda baja la más lesionada, siendo necesaria la recomendación de acciones preventivas como las posturas correctas y los esfuerzos físicos controlados con el fin de reducir los riesgos biomecánicos (Bedoya et al., 2017e; Jellad et al., 2013), existe evidencia de la recomendación de programas de educación sobre estrategias de prevención y afrontamiento para trastornos musculoesqueléticos para personal asistencial (Robstad et al., 2014). De acuerdo con Ghilan et al. (2013), tres de cada 10 enfermeras padecen dolor lumbar siendo incapacitadas en el último año, existiendo una asociación significativa entre el dolor lumbar padecido por trabajadores de enfermería y variables como la edad, la nacionalidad, los trastornos menstruales y el nivel de estrés en el trabajo. En un estudio afin a lo anterior realizado por Soroush 
et al. (2018) se hallaron tasas de prevalencia de trastornos que afectan a personal asistencial entre el $17.5 \%$ y $60.98 \%$, con molestias generadas en espalda, cuello, rodillas, parte superior de la espalda, tobillos, hombros, Manos, caderas, muslos y codos. Representando altos costos de atención médica, ausentismo en el trabajo o incluso desempleo.

Arsalani et al. (2014) señala que altas demandas de trabajo físico y psicosocial junto con un bajo control sobre el trabajo que incrementan estrés laboral aumentan considerablemente el riesgo de afecciones osteomusculares entre el personal de enfermería en tanto en países desarrollados como e aquellos en vía de desarrollo, aun en contextos sociocultural diferentes. Teniendo en cuenta lo descrito anteriormente, es determinante establecer asociaciones para evidenciar problemas osteomusculares de origen ocupacional en trabajadores del sector hospitalario, pero para ello las estadísticas descriptivas comunes en la mayoría de este tipo de trabajos se queda corta, así lo explican los autores como Gómez y Severiche (2018), Bustamante et al. (2017) y Castrillón et al. (2017), por lo cual en esta investigación se acude a la estadística inferencial de tipo multivariada, en detalle lo realizado se sintetiza en la aplicación de Modelos Logits para determinar qué variables independientes explican el riesgo de padecer problemas asociados al aparato osteomuscular.

\section{MATERIALES Y METODOS}

La población corresponde a 123 trabajadores de una clínica, para los cuales se realizó un censo muy parecido a lo trabajado por Bedoya et al. (2017). Se aplicó un instrumento a los trabajadores, donde se midieron variables asociadas a condiciones sociodemográficas, laborales, y condiciones de salud asociadas a problemas osteomusculares como se ilustra a continuación en la Tabla 1 y con base al estudio hecho por Fajardo et al. (2015):

Tabla 1: Operacionalización de las variables.

\begin{tabular}{|c|c|c|}
\hline Variable & Tipo de variable & Variable \\
\hline \multirow{2}{*}{ Características Demográficas } & \multirow{2}{*}{ Independiente } & Edad \\
\hline & & Genero \\
\hline \multirow{4}{*}{$\begin{array}{l}\text { Características asociadas a condiciones } \\
\text { laborales }\end{array}$} & \multirow{4}{*}{ Independiente } & Cargo \\
\hline & & Antigüedad laboral \\
\hline & & Recesos durante la jornada laboral \\
\hline & & Oficios por fuera del trabajo \\
\hline $\begin{array}{l}\text { Condiciones de salud asociadas al aparato } \\
\text { osteomuscular }\end{array}$ & Dependiente & Problemas osteomusculares \\
\hline
\end{tabular}

Para la información recolectada y de acuerdo con la naturaleza de las variables asociadas a condiciones demográficas, condiciones laborales y problemas osteomusculares, se realizaron los siguientes análisis estadísticos, siguiendo los lineamientos establecidos por Penna (2006): (i) Estadísticas descriptivas y (ii) Modelo Logit.

Estadísticas descriptivas. Se calculan los totales y porcentajes para las modalidades de las distintas variables, además de un diagrama de Pareto de los problemas osteomusculares que que comprende la variable dependiente para identificar los principales problemas osteomusculares que presentan los empleados.

Modelo Logit. Se ajusta un modelo Logit para determinar las variables demográficas y de condiciones de laborales que están asociadas a la presencia problemas osteomusculares. Los modelos Logit son modelos de regresión cuya variable respuesta es de naturaleza cuantitativa (Moscote y Rincón, 2012), tal como es el caso del presente estudio, donde la variable respuesta corresponde a la presencia o ausencia de problemas osteomusculares. Los análisis estadísticos se realizan en el programa estadístico R 3.15 y la hoja de cálculo Excel versión 10.

\section{RESULTADOS y DISCUSIÓN}

La Tabla 2 muestra los porcentajes obtenidos para las distintas variables demográficas, laborales y problemas osteomusculares. 
Tabla 2: Estadísticas descriptivas para las variables estudiadas

\begin{tabular}{|c|c|c|c|}
\hline Variable & Modalidad & Total & Porcentaje \\
\hline \multirow{4}{*}{ Edad } & $<30$ & 58 & $47.15 \%$ \\
\hline & $30-40$ & 41 & $33.33 \%$ \\
\hline & $40-50$ & 15 & $12.20 \%$ \\
\hline & $>50$ & 9 & $7.32 \%$ \\
\hline \multirow{2}{*}{ Genero } & Femenino & 88 & $71.50 \%$ \\
\hline & Masculino & 35 & $28.50 \%$ \\
\hline \multirow{6}{*}{ Cargo } & Auxiliar & 64 & $52.00 \%$ \\
\hline & Conductor & 5 & $4.10 \%$ \\
\hline & Coordinador & 14 & $11.40 \%$ \\
\hline & Doctor & 16 & $13.00 \%$ \\
\hline & Enfermero & 8 & $6.50 \%$ \\
\hline & $\begin{array}{l}\text { Servicios } \\
\text { Generales }\end{array}$ & 16 & $13.00 \%$ \\
\hline \multirow{2}{*}{ Recesos } & No & 54 & $43.90 \%$ \\
\hline & $\mathrm{Si}$ & 69 & $56.10 \%$ \\
\hline \multirow{2}{*}{$\begin{array}{l}\text { Enfermedad } \\
\text { Laboral }\end{array}$} & No & 113 & $91.90 \%$ \\
\hline & Si & 10 & $8.10 \%$ \\
\hline \multirow{2}{*}{$\begin{array}{l}\text { Oficios por fuera } \\
\text { del trabajo }\end{array}$} & No & 36 & $29.30 \%$ \\
\hline & $\mathrm{Si}$ & 87 & $70.70 \%$ \\
\hline \multirow{3}{*}{ Antigüedad Laboral } & $<20$ meses & 62 & $50.41 \%$ \\
\hline & $\begin{array}{l}20 \text { meses- } 40 \\
\text { meses }\end{array}$ & 49 & $39.84 \%$ \\
\hline & $>40$ meses & 12 & $9.76 \%$ \\
\hline \multirow{2}{*}{$\begin{array}{c}\text { Problemas } \\
\text { osteomusculares }\end{array}$} & No & 37 & $30.10 \%$ \\
\hline & Si & 86 & $69.90 \%$ \\
\hline
\end{tabular}

Con respecto a las variables sociodemográficas, se tiene que un importante porcentaje $(41,15 \%)$ de empleados presentan una edad inferior a 30 años; mientas que sólo un 7,32\% de los empleados presentan edades superiores a 50 años. Además, se tiene que un $71,5 \%$ de los trabajadores son de género femenino y en menor proporción se encuentra el género masculino (28,5\%). En las condiciones laborales, se observa que el cargo más frecuente es el de auxiliar, donde el $52 \%$ de los empleados poseen dicho cargo, seguido por los cargos de doctor y coordinador ( $13 \%$ y $11,4 \%$, respectivamente); mientras que sólo un $6,5 \%$ y un $4,1 \%$ presentan respectivamente, labores de enfermero y conductor.

En relación a los recesos laborales, un 43,9\% de los empleados manifiesta no realizar ningún tipo de receso laboral durante la realización de sus actividades laborales, cual según lo descrito por Guerrero et al (2004), los recesos en el trabajo influyen en el comportamiento en el trabajo y en la salud de los empleados. De acuerdo con la antigüedad laboral, el 50,41\% tiene menos de 20 meses en el trabajo; mientras que sólo un $9,76 \%$ tienen más de 40 meses. Para las enfermedades laborales, sólo un $8,1 \%$ de los empleados manifiestan haber tenido algún tipo de enfermedad laboral, pero un $69,9 \%$ indican que tienen algún tipo de problema osteomuscular.

A continuación, se muestra la distribución de problemas osteomusculares en los trabajadores. De acuerdo con Lowe et al. (2017) para las personas en edad laboral con cualquier afección musculoesquelética puede verse aumentada conforme a la carga laboral y el uso de los servicios de salud y atención médica autoinformada. Como ninguna condición musculoesquelética parece empeorar constantemente en presencia de multimorbilidad, es necesario comprender e identificar mejor las estrategias que reconocen y abordan la carga adicional de las condiciones concomitantes para los adultos en edad laboral con una variedad de condiciones musculoesqueléticas. 


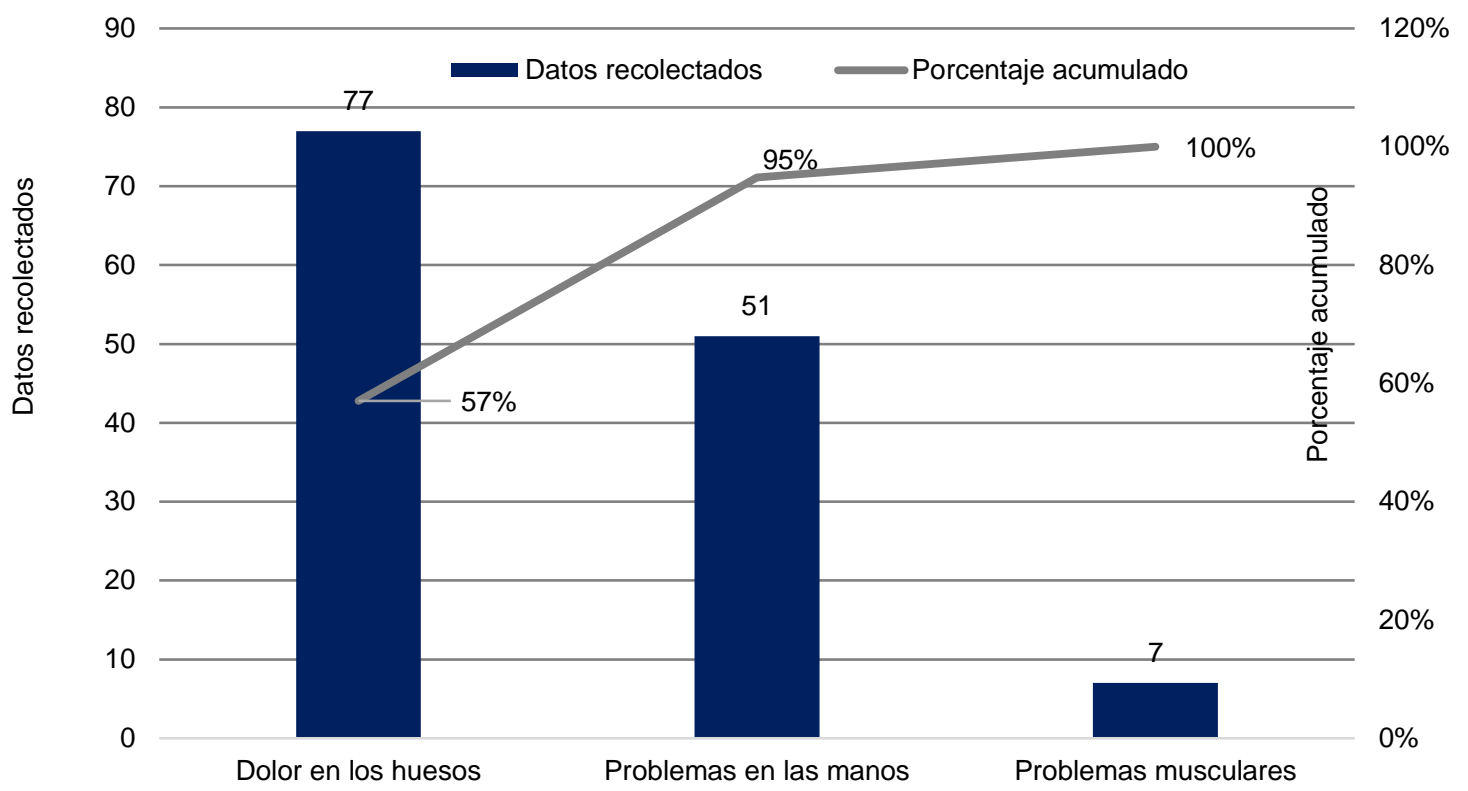

Fig. 1: Diagrama de Pareto para los problemas de salud de los empleados asociados al aparato osteomuscular

De acuerdo con la Figura 1, se observa que el principal problema que aqueja a los empleados es dolor en los huesos (77:62,6\%); seguido de problemas en las manos (51:43,09\%) y problemas musculares (7:5,69\%). La Tabla 3 muestra el modelo Logit para los problemas osteomusculares en función de factores sociodemográficos y labolares. Normalmente los trabajadores de enfermería presentan una alta prevalencia a problemas osteomusculares, según Moreira et al. (2014), esto se asocia fuertemente con la presencia de síntomas en varias regiones del cuerpo. Estos resultados indican la necesidad de programas preventivos en el entorno hospitalario para controlarlos.

Tabla 3: Modelo Logit para los problemas osteomusculares (*: Significativo ( $p$-valor $<0,1)$; ** : Altamente significativo ( $p$-valor $<0,01))$

\begin{tabular}{|c|c|c|c|c|}
\hline Variable & Beta & Exp (Beta) & $\begin{array}{c}\text { Chi-cuadrado } \\
\text { de Wald }\end{array}$ & Valor $p$ \\
\hline (Intersección) & 1.883 & 6.5724 & 1.121 & 0.290 \\
\hline [Genero=Femenino] & 1.412 & 0.2437 & 8.563 & $0.003^{* *}$ \\
\hline [Cargo=Auxiliar] & -0.625 & 0.5352 & 0.667 & 0.414 \\
\hline [Cargo=Conductor] & 1.186 & 3.2744 & 0.711 & 0.399 \\
\hline [Cargo=Coordinador] & -1.972 & 0.1392 & 3.027 & $0.081^{*}$ \\
\hline [Cargo=Doctor] & -0.261 & 0.7701 & 0.083 & 0.774 \\
\hline [Cargo=Enfermero] & -0.502 & 0.6054 & 0.200 & 0.655 \\
\hline [Recesos=No] & -0.381 & 0.6830 & 0.676 & 0.411 \\
\hline [Enfermedad Laboral=No] & -0.428 & 0.6517 & 0.271 & 0.602 \\
\hline [Oficios=No] & 0.194 & 1.2137 & 0.153 & 0.696 \\
\hline Edad & -0.009 & 0.9906 & 0.116 & 0.733 \\
\hline Antigüedad & -0.023 & 0.9771 & 2.923 & $0.087^{*}$ \\
\hline
\end{tabular}

De acuerdo con los resultados del modelo Logit, se observa que el género, cargo y la antigüedad laboral son los factores que presentan un efecto significativo en los problemas osteomusculares de los pacientes. Para el cargo de coordinador y la antigüedad laboral, se observan coeficientes negativos $(-1.972$ y -0.023 , respectivamente); mientras que para el género femenino hubo un coeficiente positivo de1. 412. Lo cual indica que los problemas osteomusculares están más asociados a empleados del género femenino y menos asociadas a empleados con labores de coordinador y de menor edad. A continuación, se muestran los diagramas de mosaicos que explican estos comportamientos. 


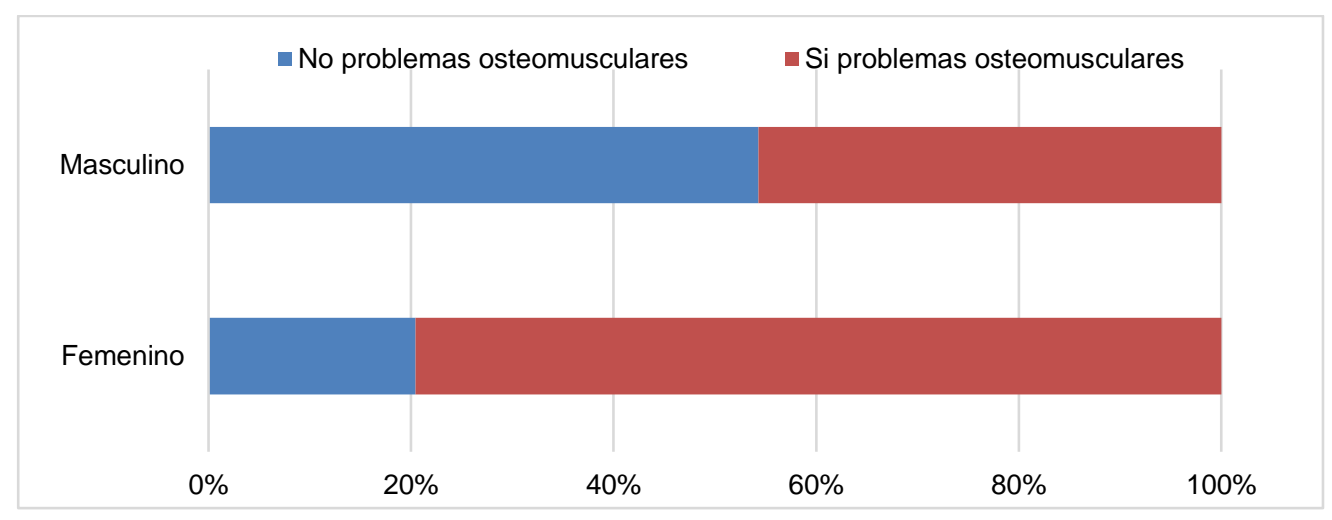

Fig. 2: Gráfico de Mosaicos entre el género y problemas osteomusculares.

En la Figura 2 se muestra el comportamiento entre el género y problemas osteomusculares mediante un gráfico de mosaicos, donde se puede observar que el género femenino presenta una proporción más grande de empleados con problemas osteomusculares.

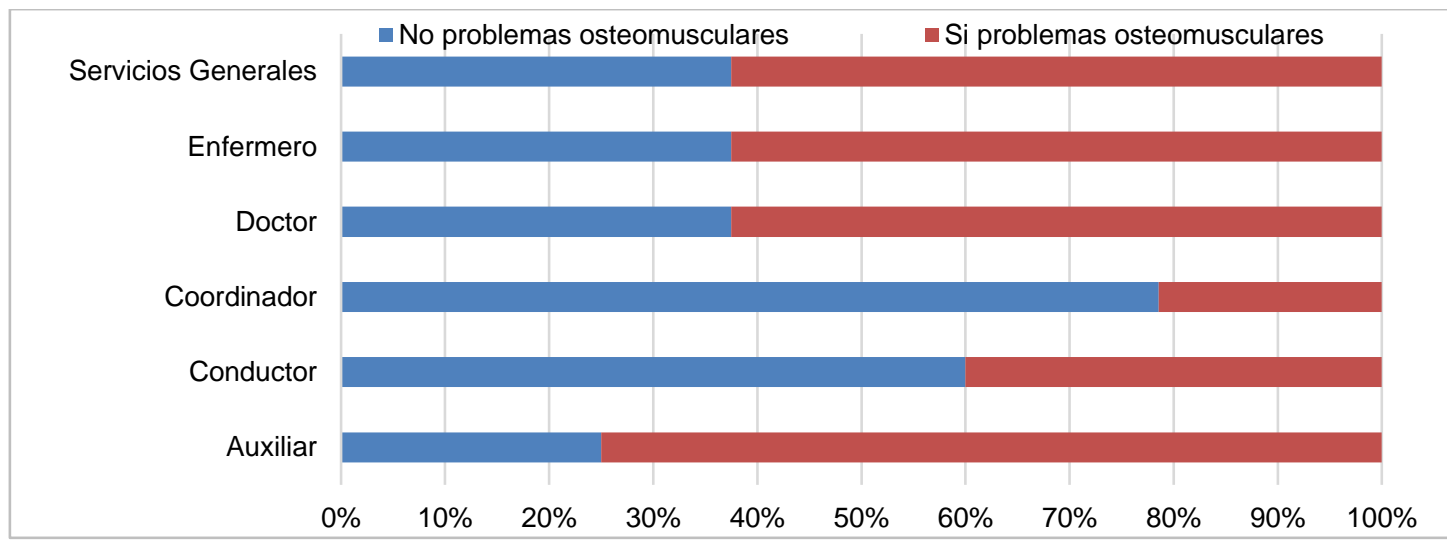

Fig. 3: Gráfico de Mosaicos entre el cargo y problemas osteomusculares.

Para el cargo, se pude ver que los que poseen labores de coordinación muestra menor porcentaje de trabajadores con problemas osteomusculares; mientras que los que tienen cargo de auxiliar muestran proporciones más altas.

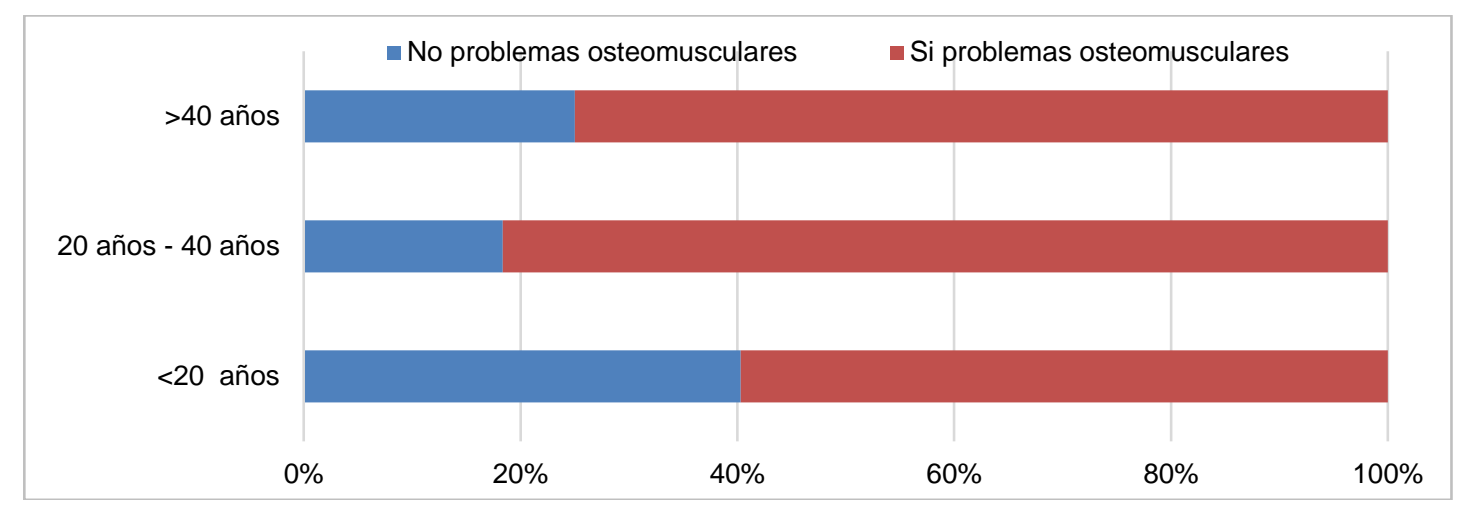

Fig. 4: Gráfico de Mosaicos entre antigüedad laboral y problemas osteomusculares.

Para la variable antigüedad laboral, se muestra en la Figura 3 que la proporción de empleados que no presenta problemas osteomusculares se encuentra en los menores de 20 años.

\section{CONCLUSIONES}

De los resultados presentados, de su discusión y de los antecedentes de la literatura expuestos a través del artículo, se pueden obtener las siguientes conclusiones principales: (i) La descripción demográfica y laboral indica que proporción de empelados más importante corresponde al género masculino, con edad inferior a 30 años, cargo de auxiliar, con una antigüedad laboral inferior a 20 meses, realizan trabajos tediosos por fuera de sus trabajos. (ii) El diagrama de Pareto muestra que los principales problemas osteomusculares 
que presentan los trabajadores son dolor en los huesos y problemas en las manos (iii) El Modelo Logit muestran que el género, la antigüedad laboral y el cargo influye son factores que pueden influir en la presencia de problemas osteomusculares (iv) Los coeficientes del modelo Logit y los diagramas de mosaico muestran que los trabajadores de género femenino tienden más que los de masculino a presentar problemas osteomusculares; mientras que los trabajadores con cargo de coordinador y menores de 20 años presentan menos en menor proporción problemas osteomusculares.

\section{AGRADECIMIENTOS}

A la Fundación Universitaria Tecnológico Comfenalco - Cartagena por el soporte financiero a través de la Dirección de Investigaciones a cargo de la Doctora Ganiveth María Manjarrez Paba, mediante la convocatoria interna de financiación de proyectos del año 2016, por el apoyo técnico de don Darío David Sierra Calderón. A don Mejía Daguer como Director del Programa de Seguridad e Higiene Ocupacional y su par para el programa de Ingeniería Industrial, don José Manuel Solana Garzón. Un especial agradecimiento por toda la logística brindada al Decano de la Facultad de Ingeniería, Doctor Ramiro Barragán Bohórquez. Por último, el trabajo operativo y de campo logrado por don Jhon Jaider Alemán Reyes y doña Mayra Alejandra Maza Pérez.

\section{REFERENCIAS}

Afshari, D., A., Mohamadi y otros dos autores, Continuous monitoring of back postures using portable inclinometer among nursing assistants, Iran Occupational Health, 11(3), 30-39 (2014)

Alperovitch-Najenson, D., I. Treger y L. Kalichman, Physical therapists versus nurses in a rehabilitation hospital: Comparing prevalence of work-related musculoskeletal complaints and working conditions, Archives of Environmental and Occupational Health, 69(1), 33-39 (2014)

Arsalani, N., M. Fallahi-Khoshknab y otros dos autores, musculoskeletal disorders and working conditions among Iranian nursing personnel, International Journal of Occupational Safety and Ergonomics, 20(4), 671-680 (2014)

Bedoya, E., C. Severiche y otros dos autores, Accidentalidad Laboral en el Sector de la Construcción: el Caso del Distrito de Cartagena de indias (Colombia), Periodo 2014-2016, Información Tecnológica, 29(1), 193-200 (2018a)

Bedoya, E.A.M., de J. Meza-Aleman y otros dos autores, Analysis of Accidentality in an Industrial Sector Company between the Years 2016-2017, International Journal of Applied Engineering Research, 13(9), 6952 - 6958 (2018b)

Bedoya, E.A., C.A. Severiche-Sierra y otros tres autores, Exposure to Industrial Noise in a Naval Maintenance Workshop of the Colombian Caribbean, International Journal of Applied Engineering Research, 13(9), 6959 - 6964 (2018c)

Bedoya, E.A., D.D. Sierra-Calderon y otros cinco autores, Labor Accidents in the Civil Works Sector Colombian Caribbean Context, International Journal of Applied Engineering Research, 13(11), 8959 - 8963 (2018d)

Bedoya, E., D. Sierra y otros dos autores, Diagnóstico de Bioseguridad en el Sector Sanitario del Departamento de Bolívar, Norte de Colombia, Información Tecnológica, 28(5), 225-232 (2017a)

Bedoya, E.A., J. Jaimes-Morales y otros dos autores, Occupational Chemical Exposure in the Urgency of a Hospital Area Public of Cartagena de indias (Colombia), International Journal of ChemTech Research, 10(10), $572-578$ (2017b)

Bedoya, E.A. M. de J. Meza-Alemán y otros dos autores, Accidentes laborales y enfermedades ocupacionales en Personal de odontología de Cartagena y departamento de Bolívar-Colombia, Ciencia Odontológica, 14(2), 21 - 26 (2017c)

Bedoya, E.A., C.A. Severiche-Sierra y otros cuatro autores, Conditions of Work and Dermatitis in Workers Exposed to Chemical Risks by Cement, International Journal of Applied Engineering Research, 12(12), 12119-12126 (2017d)

Bedoya, E.A., L. Vargas-Ortiz y otros dos autores, Kruskal-Wallis Test for the Identification of Factors that Influence the Perception of Accidents in Workers in the Construction Sector, International Journal of Applied Engineering Research, 12(17), 6730 - 6734 (2017e)

Bustamante, M., M. Lapo y otros dos autores, Factores Socioeconómicos de la Calidad de Vida de los Adultos Mayores en la Provincia de Guayas, Ecuador, Información Tecnológica, 28(5), 165-176 (2017)

Burgos, Y., E.A. Bedoya-Marrugo y otros tres autores, Chi-Square Test to Identify Factors Associated with Health Conditions in Infants of a Colombian Caribbean Root Zone, International Journal of Applied Engineering Research, 13(6), 3574-3578 (2018)

Castrillón, O., W. Sarache y E. Castaño, Sistema Bayesiano para la Predicción de la Diabetes. Información Tecnológica, 28(6), 161-168 (2017)

Dlungwane, T., A. Voce y S. Knight, Prevalence and factors associated with low back pain among nurses at a regional hospital in kwazulu-natal, south africa, doi:10.4102/hsag.v23i0.1082, Health SA Gesondheid, 23 (2018)

Fajardo, A., Trastornos osteomusculares en auxiliares de enfermería en la unidad de cuidados intensivos, Ciencia \& Trabajo, 17(53), 150-153 (2015) 
Ghilan, K., A. Al-Taiar y otros cuatro autores, Low back pain among female nurses in yemen, International Journal of Occupational Medicine and Environmental Health, 26(4), 605-614 (2013)

Gomez, E. y C. Severiche, Association between sociodemographic, Health and Work conditions in Waste pickers in Cartagena de indias (Colombian Caribbean), International Journal of Chemtech Research, 11(5), 331-341 (2018)

Guimarães, AL. y V. Felli, Notification of health problems among nursing workers in university hospitals, Revista Brasileira De Enfermagem, 69(3), 507-514 (2016)

Harcombe, H., GP. Herbison y otros dos autores, Musculoskeletal disorders among nurses compared with two other occupational groups, Occupational Medicine (Oxford, England), 64(8), 601-607 (2014)

Homaid, M., D. Abdelmoety y otros cinco autores, Prevalence and risk factors of low back pain among operation room staff at a tertiary care center, makkah, saudi arabia: A cross-sectional study, Annals of Occupational and Environmental Medicine, 28(1) 1-8 (2016)

Jellad, A., H. Lajili y otros dos autores, Musculoskeletal disorders among tunisian hospital staff: Prevalence and risk factors, Egyptian Rheumatologist, 35(2), 59-63 (2013)

Lowe, D., M. Taylor y S. Hill, Associations between multimorbidity and additional burden for working-age adults with specific forms of musculoskeletal conditions: a cross-sectional study, BMC Musculoskeletal Disorders, 18, 135 (2017)

Moreira, R., T. Sato y otros tres autores, Prevalence of musculoskeletal symptoms in hospital nurse technicians and licensed practical nurses: associations with demographic factors, Brazilian Journal of Physical Therapy, 18(4), 323-33 (2014)

Moscote, O. y W. Rincón, Modelo Logit y Probit: un caso de aplicación, Comunicaciones en Estadística, 5(2), 123133 (2012)

Munabi, I., W. Buwembo y otros tres autores, Musculoskeletal disorder risk factors among nursing professionals in low resource settings: A cross-sectional study in uganda. BMC Nursing, 13(1) (2014)

Nodooshan, H.S., A. Choobineh y otros dos autores, Ergonomic evaluation of client transfer between bed and stretcher through common way and mechanical patient transfer aid device, Iran Occupational Health, 13(5), 1-10 (2016)

Nourollahi, M., D. Afshari y I. Dianat, Awkward trunk postures and their relationship with low back pain in hospital nurses, Work, 59(3), 317-323 (2018)

Penna, F., Modelo de Regresión Logística aplicada a niños con maloclusión dental, Fundamentos en Humanidades, 11 (22), 201-211 (2010)

Robstad, AG., y RH. Westgaard, Perceived occupational exposures of home care workers and the association to general tension, shoulder-neck and low back pain, Work, 49(4), 723-733 (2014)

Soroush A., M. Shamsi y otros cuatro autores, Musculoskeletal Disorders as Common Problems among Iranian Nurses: A Systematic Review and Meta-analysis Study, Int. J. Prev. Med., 9(1), 1-9 (2018)

Szeto, G.P.Y., KT. Wong y otros dos autores, A study of spinal kinematics in community nurses performing nursing tasks, International Journal of Industrial Ergonomics, 43(3), 203-209 (2013)

Wang, J., Y. Cui y otros cinco autores, Work-related musculoskeletal disorders and risk factors among chinese medical staff of obstetrics and gynecology, International Journal of Environmental Research and Public Health, 14(6) (2017)

Wang, S.Y., L.C, Liu y otros dos autores, Comparisons of musculoskeletal disorders among ten different medical professions in taiwan: A nationwide, population-based study, PLoS ONE, 10(4) (2015)

Weiner, C., D, Alperovitch-Najenson y otros dos autores, Prevention of nurses' work-related musculoskeletal disorders resulting from repositioning patients in bed: Comprehensive narrative review, Workplace Health and Safety, 63(5), 226232 (2015)

Yan, P., F. Li y otros cinco autores, Prevalence of Work-Related Musculoskeletal Disorders in the Nurses Working in Hospitals of Xinjiang Uygur Autonomous Region, Pain Research and Management, Article ID 5757108 (2017)

Yasobant, S. y P. Rajkumar, Work-related musculoskeletal disorders among health care professionals: A cross-sectional assessment of risk factors in a tertiary hospital, India, Indian Journal of Occupational and Environmental Medicine, 18(2), 75-81 (2014)

Yusoff, H.M., R.A. Zawawi y B.M. Deros, Prevalence of lower limb pain and its associated factors among healthcare workers. Journal of Mechanical Engineering and Sciences, 11(3), 2930-2940 (2017) 